\title{
Generation of Virtual WDS to Complete Cadastral Information of Existing Systems
}

\author{
Juliana Robles ${ }^{1}$, Juan Saldarriaga ${ }^{2}$ \\ ${ }^{1}$ Research Assistant, Water Supply and Sewer Systems Research Center CIACUA, Universidad de los \\ Andes, Bogota; e-mail: j.robles1812@uniandes.edu.co \\ ${ }^{2}$ Professor, Department of Civil and Environmental Engineering, Universidad de los Andes, Bogota; \\ e-mail: j-saldarr@uniandes.edu.co
}

\begin{abstract}
Information related to Water Distribution Systems (WDS) in small and intermediate cities often may be incomplete or unreliable, and data acquisition may be infeasible due to elevated time and economic costs. This lack of information can lead to inaccurate network operation and maintenance, and it can also limit the use of calibrated hydraulic models to support future designs in the system. Virtual case studies can thus be treated and used to overcome the insufficient data problems. The results are not case-specific as the obtained with benchmark networks. This paper aims to evaluate a new methodology by generating virtual networks for different cities in Colombia and then comparing resulting virtual WDS with the real systems, based on data obtained from water utilities. For both sets, network layout similarity and hydraulic factors were analysed. The results of the comparison between real and virtual networks make it possible to conclude about the applicability and limitation of this virtual generation software in small cities or DMAs in large cities, and also allows suggesting alternative solutions.
\end{abstract}

\section{INTRODUCTION}

Water Distribution Systems (WDS) are an important part of public networks in cities, as they allow the population of a service area to receive water in optimal conditions, regarding pressure and quality. Therefore, a proper performance that guarantees the accomplishment of the service levels requires an ongoing maintenance based on a detailed knowledge of the system components. This information is vital in WDS modelling when it comes to monitoring the system and plan expansions, among others. However, disorganized and rapid population growth may complicate data acquisition and adversely affect the quality of it. For water utilities, this process is costly in terms of time and money, and may achieve incomplete models only and, as a result, regular system analysis.

Research strategies include study cases to create, prove and amend theories. Benchmark networks as New York Tunnels, Two Loops, Anytown and Hanoi were developed to compare results obtained in different studies. Though these networks were extensively used with test aims, the results are case-specific and should not be applied to different scenarios (Sitzenfrei, 2012).

Due to safety reasons and poor data sets, investigations can also rely on virtual or semivirtual networks. These models tackle the lack of information and mimic real data sets development that can be used as case studies or preliminary designs for new systems. One common example of virtual networks are Micropolis and Mesopolis presented by Brumbelow, Torres, Guikema, Bristow, and Kanta (2007). This WDS include developmental timeline spanning and critical infrastructure dependencies in addition to geography, demography and 
physical elements information. Some research studies implemented on these networks include contaminant propagation analysis and mitigation strategies for urban fire events.

During the last years, various approaches were developed to automatically generate virtual WDS for research purposes. Modular System Design (MSD), Water Distribution System Designer (WDS), HydroGen and WaterNetGen are some of these algorithms, which include creating the layout and a pipe-sizing algorithm, among other features (Sitzenfrei, 2016). Several studies have tested these approaches with different advantages relying on the research aim. DynaVIBe-Web is the latest approach developed by the University of Innsbruck as an automatic generation tool based on few and available free street network data. This software is implemented in this paper to verify if the use of the generated virtual models is suitable for small municipalities in Colombia.

\section{SOFTWARE DESCRIPTION}

DynaVIBe-Web is a simplified version of the DynaVIBe approach and is available after a free online registration. This software generates virtual networks based on limited, easy accessible information. It takes into account the strong correlation between street networks and water infrastructure, using the free available Open Street Map data. Generation procedure requires the definition of a service area (free hand drawing), location of water sources and a DEM file. Additionally, input parameters include total demand, number of networks to generate, cycle indicator (CI) and mainpipe offset (MPO).

The Water Supply Network layout generation first obtains the street data inside the service area with the drawn polygon. Afterwards, the water sources are connected and the elevation of each node is set using the Digital Elevation Model. The software understands the system as a graph $(\mathrm{G}=(\mathrm{E}, \mathrm{V}))$, where edges refer to pipes and vertices to nodes. A spanning tree is calculated (random or minimum, based on user choice), which is a subgraph of $\mathrm{G}$ that includes all existing vertices to create a connected, acyclic tree. The former is obtained with greedy algorithms and the latter with Kruskal algorithm. Next, demand is approximated in the nodes depending on the user selection (uniformly or normal distributed) and the edges connecting nodes without demand are removed. As a final step, loops can be created in the network based on the CI. New edges are added as alternatively path between two vertices if its length is CI-times smaller compared with the same length in the spanning tree (Mair, Rauch, \& Sitzenfrei, 2014).

In Paez and Fillion (2016), 45 WDS were generated and compared with a set of 12 modified real networks, in terms of graph theory and hydraulic indexes. As a result, "maximum possible graph" was included in the layout options of the software due to a low connectivity result for the virtual models. This option cancels the CI value, creating as many loops as possible. Therefore, synthetic network connectivity successfully increased and the set showed a satisfactory performance for the reliability-related indexes compared to the real ones.

\section{METHODOLOGY}

In this study, a set of six WDS from small cities and DMAs in large cities of Colombia (assumed to be the exact representation of the system) were compared with virtual networks generated in DynaVIBe-Web. The comparison included graph theory and hydraulic indexes to verify the 
accuracy of the synthetic models. Additionally, the possibility of using the virtual model to complete cadastral information was evaluated including known data from the already existing network. Regarding this, the methodology is described below using as example one real network from the data set.

\section{Virtual network generation}

The example network is a small city of Colombia. According to the EPANET model provided by the water utility, the WDS has 838 nodes, 710 pipes and 2 reservoirs. The total length of the system is $47.59 \mathrm{~km}$ with a demand of $75.76 \mathrm{~L} / \mathrm{s}$. In order to generate a virtual network as similar as possible to the existing one, the MPO was calculated by creating an offset for the WDS in ArcGIS. However, the study noticed the street network data from Open Street Map (OSM) for the municipality was not updated. Using an OSM editor, 39 new streets were added based on available aerial photos. With the resulting model, Figure 1 presents in aquamarine color the main system and the performed offset in colors. As the MPO value defines the location of the biggest pipes on the border of the polygon, different distances were tried to find the option with the highest number of pipes above $150 \mathrm{~mm}$ in the same location of the real network.
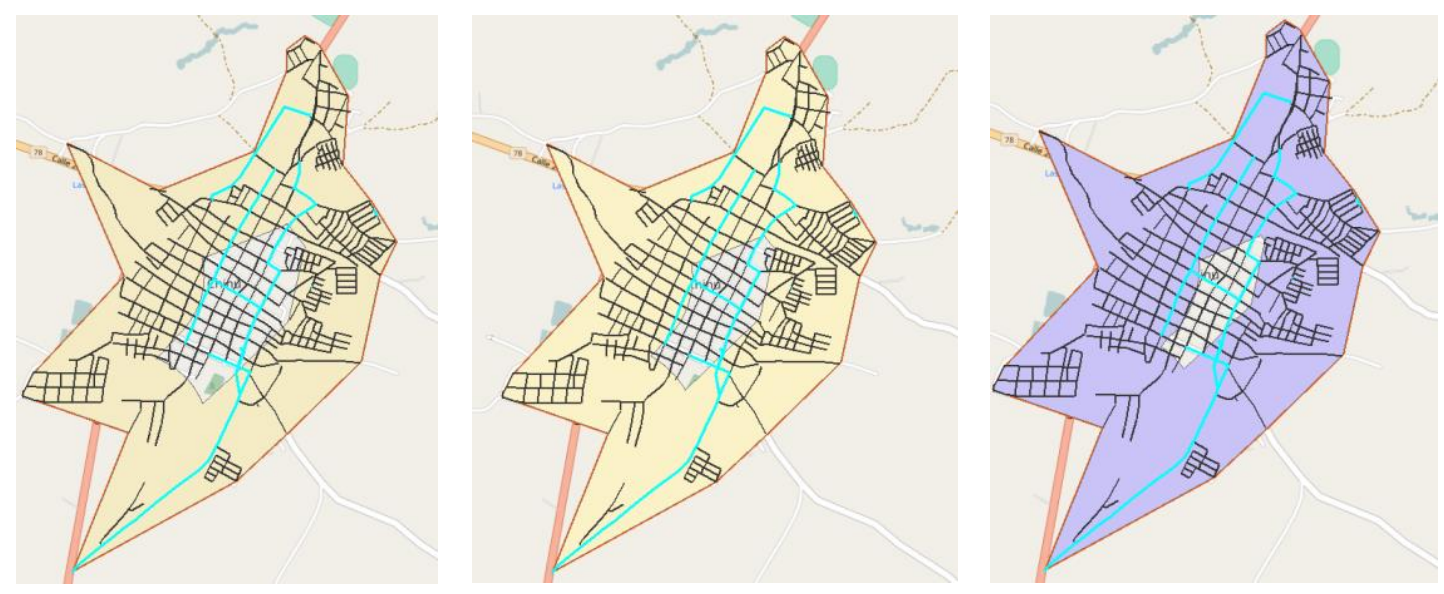

Figure 1. Offset of the service polygon for: $450 \mathrm{~m}$. (left), b $500 \mathrm{~m}$. (middle), c. $550 \mathrm{~m}$. (right)

As noticed, the offset methodology hardly represents the location of the main system. Consequently, the highest number of pipes placed in the same location was 14 for case $b$. A further analysis was developed 510 and $540 \mathrm{~m}$ and the results were 16 and 24 for 510 and 540 $\mathrm{m}$, respectively. Therefore, the selected MPO for this network was $540 \mathrm{~m}$ which best fitted the expected design, correctly representing $30 \%$ of the main pipes (diameter above $150 \mathrm{~mm}$ ). 

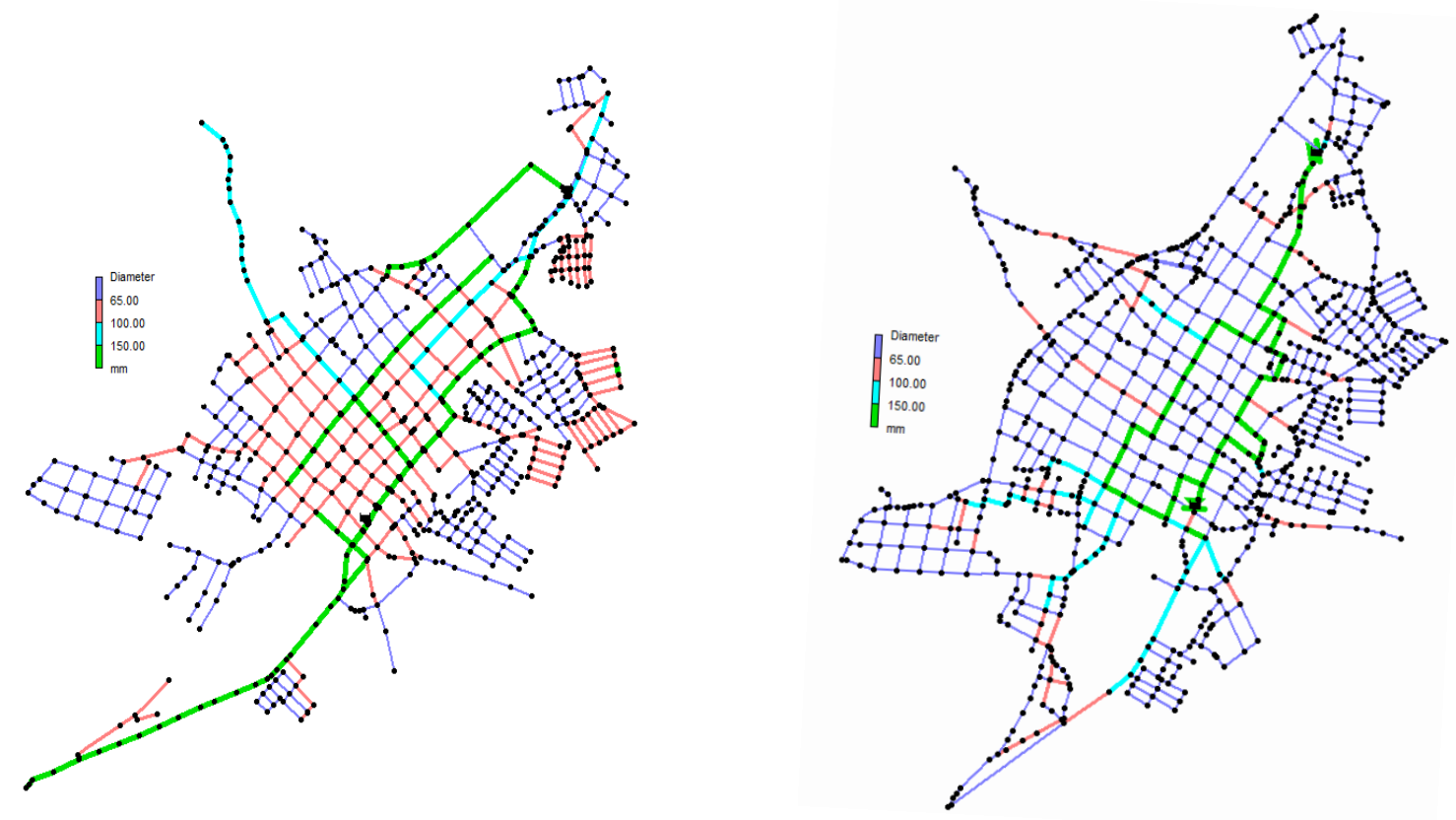

Figure 2. Real network (left) and virtual network with diameter distribution

\section{Graph theory indexes}

Water distribution networks can be studied as a complex network using measurements that quantify connectivity and structure. Several studies were achieved taking into account this approach and the results enabled the organization and reliability analysis of this infrastructure, as well as its comparison with other networks. WDS is treated as an undirected graph modelling all junctions and sources as vertices and links, pumps and valves as edges. In this research, graph theory metrics were used to compare real and virtual networks in terms of structure, efficiency and robustness.

Description of the structural indices used are presented in Table 1. For the example network, Link Density of the real and virtual network was very similar (0.0035 - 0.004), since the number of edges and vertices was close. This is an indicator of the sparseness of the networks, due to the low values obtained (near zero).

Average Node Degree $(k)$ and Central Point Dominance $\left(c^{\prime}{ }_{b}\right)$ quantify the structural organization of a network. The first refers to the average connection number of a node in the network, usually maximum 4 in WDS. The latter metric describes how controlled the network is by central points, which tends to be close to zero regarding the typical grid structure of this systems. The values obtained for these indexes were $2.5-2.7$ for the real and virtual $k$ and 0.17 -0.15 for $\mathrm{c}_{\mathrm{b}} \mathrm{b}$.

Additionally, the Average Clustering Coefficient is a metric to quantify the loops in a network and can therefore indicate its redundancy. However, it considers the density of triangles in the network, which is not common in WDS. The low value obtained for the studied networks $(0-0.006)$ confirms this statement. For that reason, the Meshedness Coefficient quantifies path 
redundancy for all types of cycles. The obtained results $(0.12-0.17)$ evidence a slight increase in virtual networks loops with respect to the real one, suggesting an enhanced connectivity.

Table 1. Graph Theory metrics used to assess network structure

\begin{tabular}{|c|c|c|}
\hline Indicator & Description & Formula \\
\hline Link Density & $\begin{array}{l}\text { Fraction between total and maximum possible edges of a } \\
\text { network } \\
\text { (Yazdani and Jeffrey, 2001; Paez and Fillion,2016) }\end{array}$ & $q=\frac{2 e}{v(v-1)}$ \\
\hline $\begin{array}{c}\text { Average } \\
\text { Node Degree }\end{array}$ & $\begin{array}{l}\text { Average number of links connected to a node } \\
\text { (Yazdani and Jeffrey, 2011) }\end{array}$ & $k=\frac{2 e}{v}$ \\
\hline $\begin{array}{l}\text { Average } \\
\text { Clustering } \\
\text { coefficient }\end{array}$ & $\begin{array}{c}\text { Measures the density of triangles in the graph, ratio of } \\
\text { actual to possible number of triangles that pass through a } \\
\text { node } \\
\text { (Yazdani and Jeffrey, 2001) }\end{array}$ & $C=\frac{3 N_{\Delta}}{N_{3}}$ \\
\hline $\begin{array}{l}\text { Meshedness } \\
\text { Coefficient }\end{array}$ & $\begin{array}{l}\text { Ratio between actual and maximum possible number of } \\
\text { loops in a planar graph } \\
\text { (Buhl et al.2006; Porse and Lund, 2016) }\end{array}$ & $R_{m}=\frac{e-v+1}{2 v-5}$ \\
\hline
\end{tabular}

Characteristic Path Length and Diameter are indicators of accessibility and connectivity (Table 2 ). The shortest path between two nodes is the minimum number of edges that must be crossed to reach from one node to another. The Characteristic Path Length $(l)$ is the average of the shortest paths, being a small value a signal of a quick transfer and high efficiency. The values obtained for the networks were 23.7 (real) and 19.2 (virtual), showing an increase in the efficiency of the synthetic model with respect to the real one. Additionally, the Diameter is calculated as the maximum shortest path. The results of $d$ were consistent with $l$, a greater diameter was obtained for the real network (79) which had a higher $l$ compared to the virtual model (50).

Table 2. Graph Theory metrics used to assess network efficiency and accessibility

\begin{tabular}{ccc}
\hline Indicator & Description & Formula \\
\hline $\begin{array}{c}\text { Characteristic } \\
\text { Path Length }\end{array}$ & $\begin{array}{c}\text { Average degree of separation between all nodes in the } \\
\text { network (Porse and Lund, 2016) }\end{array}$ & $l=\frac{1}{v(v-1)} \sum_{i \neq j} d_{i j}$ \\
\hline Diameter & $\begin{array}{c}\text { Greatest distance between a pair of vertices } \\
\text { (Yazdani and Jeffrey, 2001) }\end{array}$ & $\begin{array}{c}\text { Maximum value of the } \\
\text { shortest paths }(d)\end{array}$ \\
\hline
\end{tabular}

Finally, three measures were used to assess the robustness and vulnerability of the networks (Table 3). Density of Bridges showed a significant difference between the real and virtual model. While the former required a minimum of 206 edges (23\%) to be removed before the graph was disconnected, the latter needed 64 (6\%). Spectral Gap and Algebraic Connectivity are indexes that indicate robustness. Values calculated for $\nabla$ were close to each other 0.09 (real) -0.07 (virtual), as with the $\lambda_{2}(0.001-0.003)$. Although the virtual network presented a lower Density 
of Bridges and Spectral Gap values, it had a higher Algebraic Connectivity. The differences between calculated values are marginal so the study concluded the networks has similar robustness characteristics, consistent with literature reports.

Table 3. Graph Theory metrics used to assess network robustness and vulnerability

\begin{tabular}{ccc}
\hline Indicator & Description & Formula \\
\hline $\begin{array}{c}\text { Density of } \\
\text { Bridges }\end{array}$ & $\begin{array}{c}\text { Number of edges needed to be removed in order to } \\
\text { disconnect the graph divided between all edges } \\
\text { (Yazdani and Jeffrey, 2001; Paez and Fillion,2016) }\end{array}$ & $D_{b r}=\frac{N_{b r}}{e}$ \\
\hline $\begin{array}{c}\text { Spectral } \\
\text { Gap }\end{array}$ & $\begin{array}{c}\text { Difference between the two biggest eigenvalues of the } \\
\text { adjacency matrix of the graph } \\
\text { (Yazdani and Jeffrey, 2001; Paez and Fillion,2016) }\end{array}$ & $\nabla=\lambda_{1}(A)-\lambda_{2}(A)$ \\
\hline $\begin{array}{c}\text { Algebraic } \\
\text { Connectivity }\end{array}$ & $\begin{array}{c}\text { Second smallest eigenvalue of the Laplacian matrix of } \\
\text { the graph (Yazdani and Jeffrey, 2001; Paez and } \\
\text { Fillion,2016) }\end{array}$ & $\lambda_{2}$ \\
\hline
\end{tabular}

\section{Hydraulic indexes}

The virtual model generated for the example network had a favorable performance in terms of pressure, regarding the results of an extended period simulation with the real demand pattern. The synthetic network proved sufficient hydraulic performance considering that an adequate pressure in the alpine region ranges from 40 to $100 \mathrm{~m}$ (Sitzenfrei, Möderl, and Rauch, 2010). However, the reservoirs were placed almost 29 and $26.2 \mathrm{~m}$ above the real height to fulfill the pressure requirements. In this case, the energy of the system increased entailing that $82 \%$ of the pipes had a minimum diameter $(50 \mathrm{~mm})$ compared to a $44 \%$ in the real case. To make results comparable, roughness and reservoirs elevation were equal for both models.

Performance of the virtual networks was evaluated using pressure, power and quality indices. Minimum, mean and maximum pressure are computed along with mean pressure surplus (MPS) and mean pressure deficit (MPD), indicators of the network working condition (Di Nardo, Di Natale, Santonastaso, Tzatchkov and Alcocer-Yamanaka, 2015).

$$
\begin{aligned}
& M P S=\frac{\sum_{i=1}^{n} \propto_{i} Q_{i}}{\sum_{i=1}^{n} Q_{i}}, \text { where }\left\{\begin{array}{cc}
\alpha_{i}=0 ; & \forall i: h_{i} \leq h_{i}^{*} \\
\propto_{i}=h_{i}-h_{i}^{*} ; & \forall i: h_{i}>h_{i}^{*}
\end{array}\right. \\
& M P D=\frac{\sum_{i=1}^{n} \beta_{i} Q_{i}}{\sum_{i=1}^{n} Q_{i}}, \text { where }\left\{\begin{array}{cc}
\beta_{i}=0 ; & \forall i: h_{i} \geq h_{i}^{*} \\
\beta_{i}=h_{i}^{*}-h_{i} ; & \forall i: h_{i}>h_{i}^{*}
\end{array}\right.
\end{aligned}
$$

The pressure head surface for both networks is similar when calculated for the peak demand hour, as presented in Table 4. The results show an acceptable performance of the virtual network in terms of minimum pressure requirements $(15 \mathrm{~m})$. The MPD suggests a small deficit in the pressure service caused by the change in the elevation of the reservoir. 
Table 4. Pressure heads for the real and virtual networks

\begin{tabular}{llllll}
\hline Network & $\begin{array}{l}\text { Minimum } \\
(\mathrm{m})\end{array}$ & $\begin{array}{l}\text { Median } \\
(\mathrm{m})\end{array}$ & $\begin{array}{l}\text { Maximum } \\
(\mathrm{m})\end{array}$ & $\begin{array}{l}\text { MPS } \\
(-)\end{array}$ & $\begin{array}{l}\text { MPD } \\
(-)\end{array}$ \\
\hline Real & 6.49 & 35.24 & 21.40 & 6.94 & 0.41 \\
Virtual & 9.22 & 36.32 & 23.17 & 7.34 & 0.33
\end{tabular}

The spatial distribution difference in pressure and elevation was calculated using ArcGIS and is shown in Figure 3 (Araque and Saldarriaga, 2005). The hydraulic pressure head surface of the virtual model was subtracted from the hydraulic pressure head surface of the real system. At some points, junctions of the real set can reach up to $13.8 \mathrm{~m}$ above the virtual nodes and $5.6 \mathrm{~m}$ below in other regions. Figure 4 shows that for the $5 \%$ of the supply area, the virtual network has pressure head values between 10 and $22 \mathrm{~m}$ above the real model. Similarly, for $35 \%$ of the supply area, the virtual network has higher pressure head values and for the $9 \%$, the real model has a difference above $11 \mathrm{~m}$. The entire supply area difference values are between $\pm 23 \mathrm{~m}$.
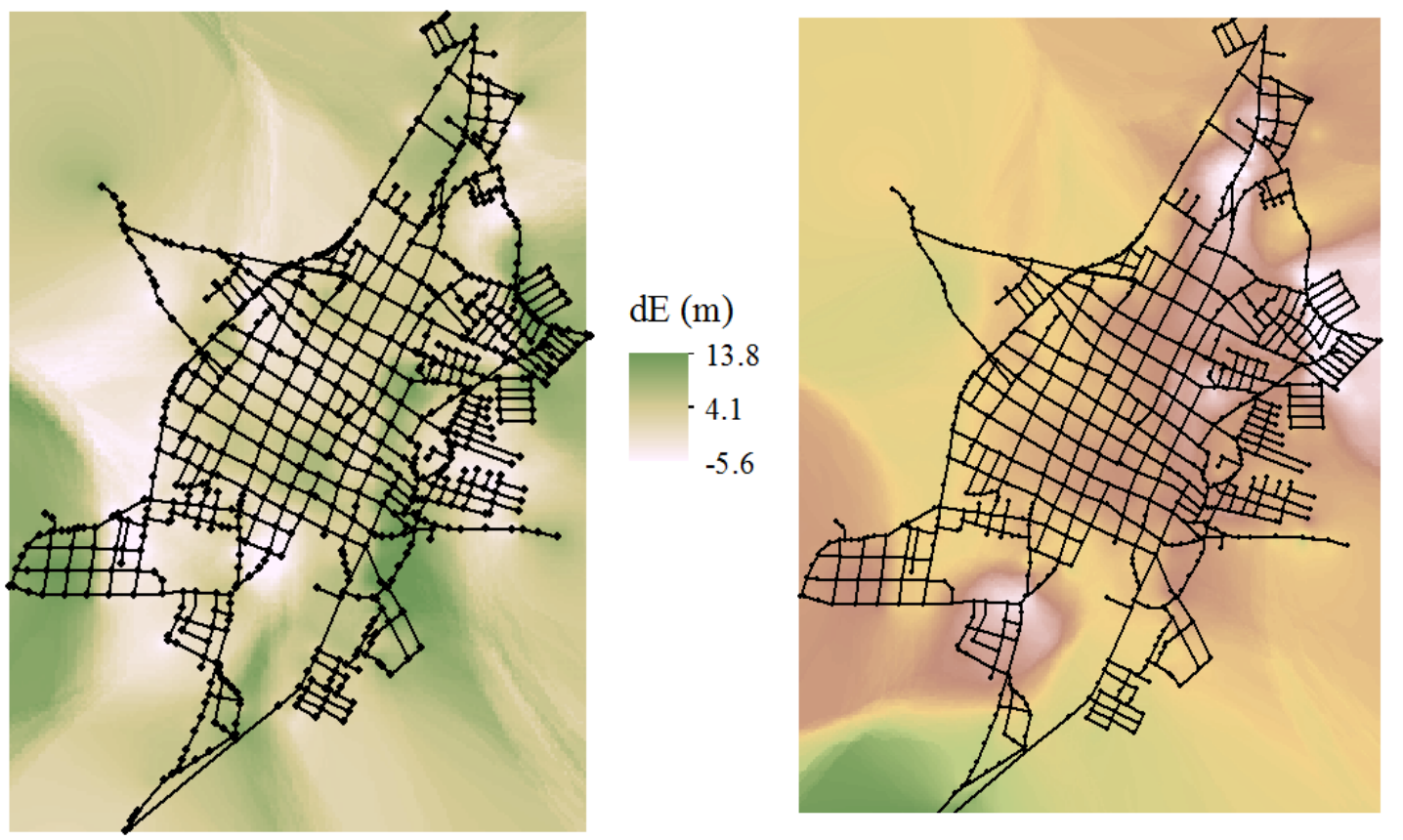

Figure 3. Difference in elevation (dE) and pressure head (dP) between the real and virtual networks 

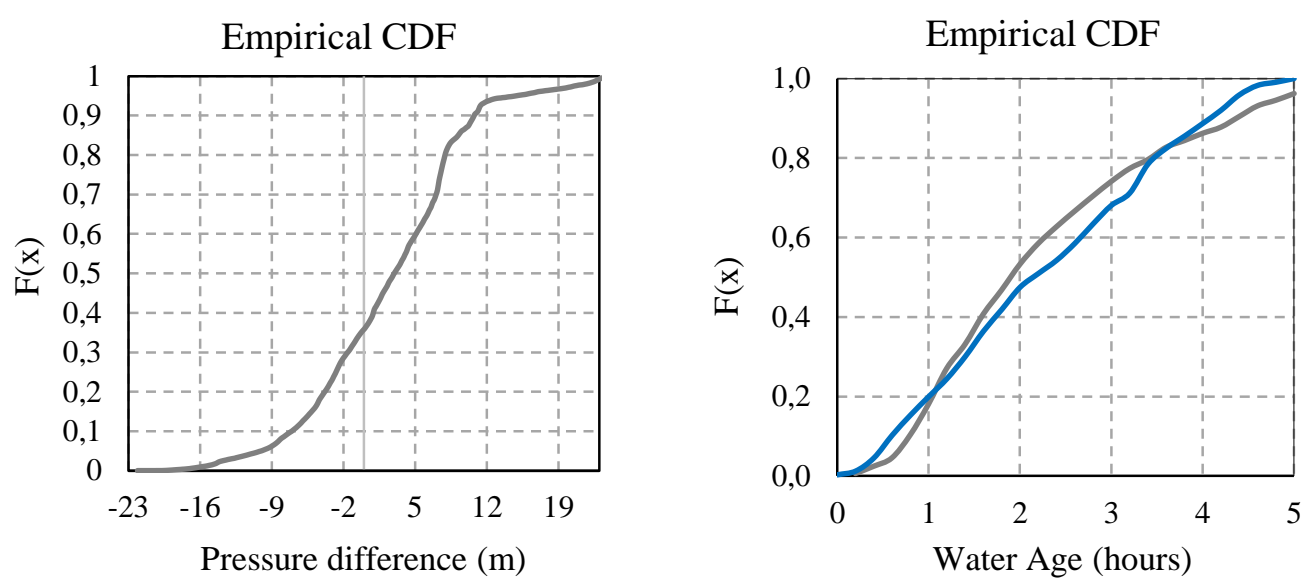

Figure 4. Hydraulic pressure head difference and water quality age CDF

The Resilience Index is described as the intrinsic capability of overcoming stress conditions (Todini, 2000) and is defined by the following expression. $D_{i}$ refers to the demand in node $i ; H_{i}^{*}$ is the required head; $H_{i}$ is the computed head in node $i ; D_{\text {out }}$ is the outflow from reservoir $k$ and $H_{k}$ is the head in the reservoir $k$ (Paez and Filion, 2016).

$$
R I=\frac{\sum_{i=1}^{n_{n}} D_{i}\left(H_{i}-H_{i}^{*}\right)}{\sum_{k=1}^{n_{r}} D_{\text {out }_{k}} H_{k}+\sum_{i=1}^{n_{n}} D_{i} H_{i}^{*}}
$$

For the example network, the value computed for the virtual and real model was 0.36 and 0.46 , respectively. The result indicates a better performance for the real system, considering a higher resilience value is related to a higher energy redundancy and lower internal energy dissipation. Additionally, results of median water age for the real and virtual systems were 2.11 and 2.35 hours respectively, with the cumulative distribution presented in Figure 4. The calculated values for power and quality analysis were close for both networks, certifying the capability of the virtual network to reproduce the real set.

\section{Completing cadastral information}

Additionally, a different virtual network was created to validate the proposed expression for MPO index. The three different indexes were calculated using ArcGIS and the best MPO option was obtained. With DynaVIBe-Web, a synthetic network was generated based on the real world model information. Afterwards, the virtual model was calibrated based on a trial and error procedure with diameters and elevation as hydraulic variables to verify the software capability to correctly represent a WDS. Results are presented below. 


\section{RESULTS}

\section{Virtual network generation}

Six networks were obtained from water utilities in Colombia; their layout is presented in Figure 5.

1.

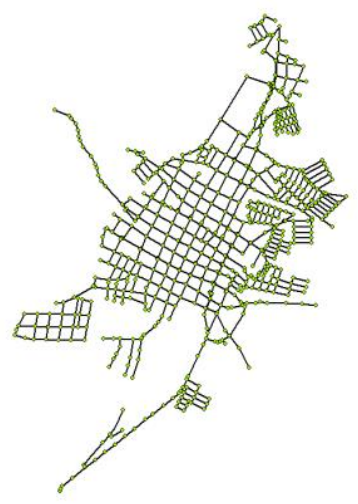

4.

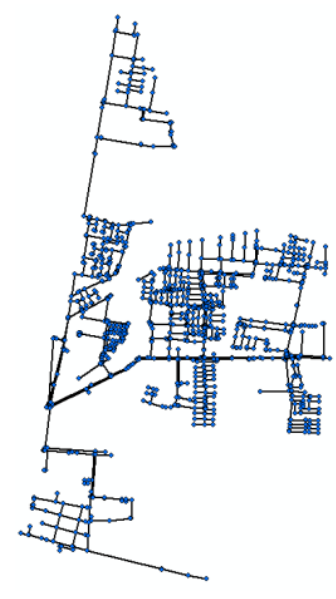

2.

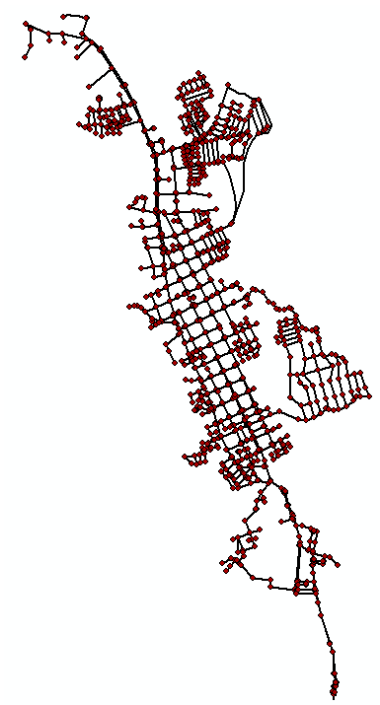

5.

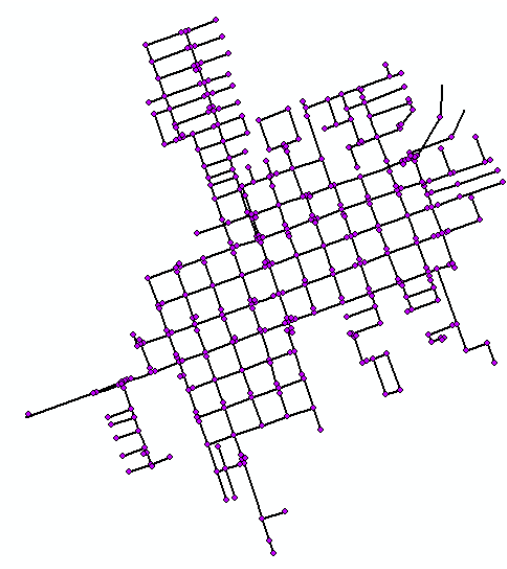

3.

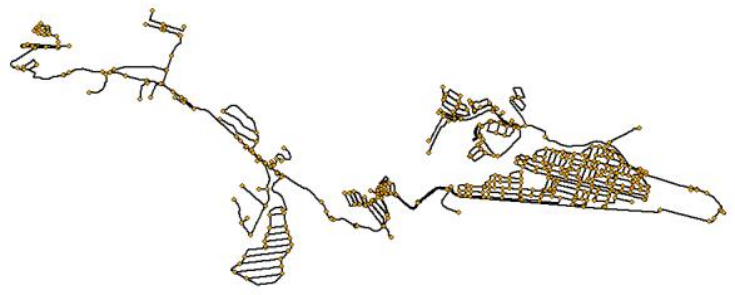

6.

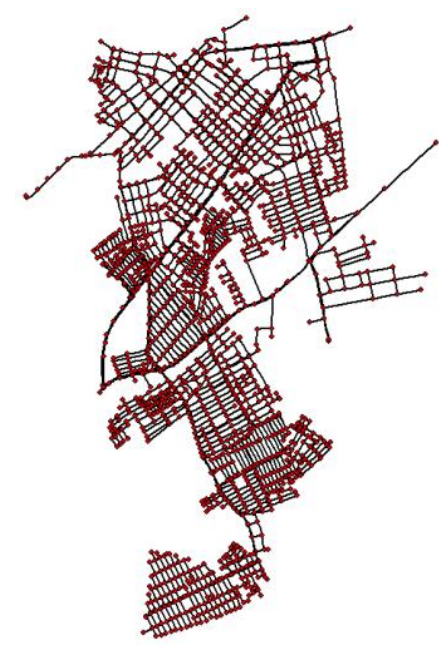

Figure 5. Layout of the study cases

Virtual networks were generated using DynaVIBe-Web based on information available for the original models. Detailed characteristics for each one is shown in Table 5. 
Table 5. Characteristics of the study cases

\begin{tabular}{cccccc}
\hline Network & Pipes & Junctions & Reservoirs & Demand (L/s) & Length $(\mathrm{km})$ \\
\hline 1 & 1092 & 1231 & 1 & 66.30 & 54.10 \\
2 & 838 & 710 & 2 & 98.50 & 47.59 \\
3 & 641 & 567 & 1 & 35.00 & 38.34 \\
4 & 2800 & 2139 & 2 & 595.41 & 166.81 \\
5 & 1291 & 1191 & 1 & 69.25 & 38.94 \\
6 & 469 & 398 & 2 & 25.06 & 18.74
\end{tabular}

Results obtained from the described methodology are presented below, including MPO values and percentage of pipes with diameter above $150 \mathrm{~mm}$ correctly placed (compared to real models).

Table 6. Best MPO obtained for the analyzed networks and correctly placed pipes (\%)

\begin{tabular}{ccc}
\hline Network & MPO $(\mathrm{m})$ & $\%$ \\
\hline 1 & 100 & $34 \%$ \\
2 & 540 & $3 \%$ \\
3 & 130 & $2 \%$ \\
4 & 130 & $20 \%$ \\
5 & 1 & $29 \%$ \\
6 & 250 & $8 \%$
\end{tabular}

The methodology described above was used to find the optimum MPO value for each network and the following expression was obtained based on the results. Three indicators were introduced considering the generation process is based on street networks data, a polygon and a reservoir(s) location. Therefore, $I_{1}$ is the ratio between the perimeter and area of the polygon, quantifying the compactness. $\mathrm{I}_{2}$ is the mean real density of streets inside the polygon and $\mathrm{I}_{3}$ refers to the maximum distance between the polygon centroid and the reservoir(s) as a measure of dispersion. The results for each indicator are shown below.

Table 7. Value of evaluated indicators

\begin{tabular}{cccc}
\hline Network & I1 & I2 & I3 \\
\hline 1 & 4.19 & 3.59 & 2.64 \\
2 & 2.81 & 8.22 & 1.16 \\
3 & 4.71 & 4.94 & 2.27 \\
4 & 1.38 & 3.90 & 1.90 \\
5 & 5.10 & 8.87 & 2.15 \\
6 & 7.50 & 7.00 & 0.58
\end{tabular}

The following expression can be used by researchers interested in using the software to generate virtual networks of small municipalities in Colombia.

$$
M P O=657.5-42.8 I_{1}+8.8 I_{2}-188.4 I_{3}
$$

Although this approach results in the best MPO value that represents a real network, the number of pipes of the main that are correctly located is very low. Hence, a different methodology should be 
implemented to improve the virtual models accuracy. This could include the possibility of drawing the main pipe path or adding a demand surface as an input.

\section{Graph theory indexes}

The number of edges and vertices of the real and virtual networks studied is presented in Table 8 . These results were computed after a skeletonization process using WaterGEMS (Saldarriaga, Ochoa, Rodríguez and Arbeláez, 2008), due to the tendency of the software to create several nodes with zero base demand, and in order to represent the real form of curve streets. Four of the six virtual networks density values were higher (less sparse) compared with the real ones. This is caused by a reduced number of edges and vertices in the model. Network 6 presented the higher difference due to its reduced number of vertices which had a major impact on the formula. However, computed values for both cases is similar and consistent with literature results. On the other hand, the analyzed networks have, as expected, node degree distribution values between 2.1 and 3.1 (uniform distribution). This, viewed in line with low c' ${ }_{b}$ values, confirm the planarity of the water distribution systems.

Table 8. Link Density values for analyzed networks

\begin{tabular}{ccc}
\hline Network & Real & Virtual \\
\hline 1 & 0.0019 & 0.0035 \\
2 & 0.0035 & 0.0037 \\
3 & 0.0041 & 0.0035 \\
4 & 0.0012 & 0.0018 \\
5 & 0.0014 & 0.0037 \\
6 & 0.0059 & 0.0208
\end{tabular}

The characteristic path length and diameter was lower in four of the six virtual models. This suggests a more compact and efficient network. However, the diameter should be analyzed regarding the size of the network due to the presence of high and low compact regions that affects the result (Porse and Lund, 2016). Figure 6 presents on the left side, the ratio of diameter value to number of network vertices. Three of the real networks resulted in lower values considering its size. Values obtained for virtual models are close to the real ones.

Density of bridges, spectral gap and algebraic connectivity measures were used for the robustness analysis. $\mathrm{D}_{\mathrm{br}}$ values for real networks are higher for five of the six networks although the difference is below $26 \%$. For the $\nabla$ index, a relative difference or the results was computed with networks 3, 4 and 5 showing a better performance while the majority of virtual models resulted in higher values of $\lambda_{2}$ (i.e. higher robustness against failures). 

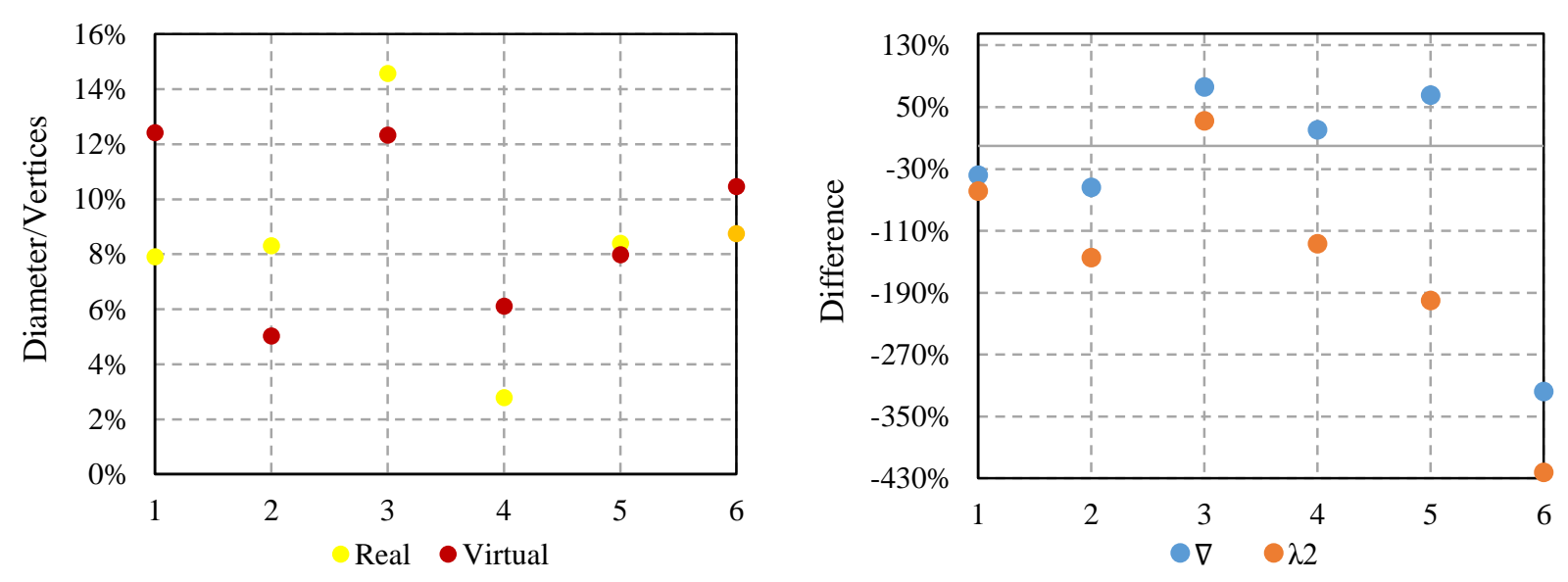

Figure 6. Differences in efficiency (left) and robustness (right) indices between real and virtual networks

\section{Hydraulic indexes}

Altering the reservoir elevation had a major impact on the virtual models. Therefore, some nodes presented very low and negative pressure values during regular and maximum demand hours. Results Table 10 for the hydraulic indices used to assess network performance during peak hour demand are presented below.

Table 9 shows network 4 and 6 had the worst working conditions with the highest mean pressure deficit values, while networks 3 and 5 had the lowest results (i.e. better performance). For network 3 , the pressure head values were almost doubled in the virtual network reaching $221 \mathrm{~m}$ vs $114 \mathrm{~m}$. The real model had several pressure control valves to maintain adequate values in the system. Maximum pressure heads for the remaining networks were similar. However, the virtual models 1, 4 and 6 presented minimum negative pressures heads in the peak hour reaching values of $22 \mathrm{~m}$ for network 1.

Table 9. Pressure indices for real and virtual networks (m)

\begin{tabular}{ccccc}
\cline { 2 - 5 } & \multicolumn{2}{c}{ Real } & \multicolumn{2}{c}{ Virtual } \\
\hline Network & MPS & MPD & MPS & MPD \\
\hline 1 & 11.66 & 1.22 & 8.95 & 5.01 \\
2 & 6.94 & 0.41 & 7.34 & 0.33 \\
3 & 31.04 & 0.00 & 90.00 & 0.01 \\
4 & 8.87 & 0.00 & 1.61 & 8.72 \\
5 & 7.12 & 1.42 & 18.32 & 0.00 \\
6 & 1.22 & 1.56 & 0.00 & 10.8
\end{tabular}

Additionally, mean water age values were similar with a maximum difference of 5 hours. Resilience index was computed for both models showing, in four of the six cases, a better performance for the real networks due to the change in the tank elevation. 
Table 10. Mean Water Age and Resilience Index values for real and virtual networks (hours)

\begin{tabular}{ccccc} 
& \multicolumn{2}{c}{ Mean Water Age (hours) } & \multicolumn{2}{c}{ Resilience Index (-) } \\
\hline Network & Real & Virtual & Real & Virtual \\
\hline 1 & 6.61 & 6.45 & $3.56 \times 10^{-4}$ & $6.97 \times 10^{-2}$ \\
2 & 3.19 & 2.84 & $4.59 \times 10^{-1}$ & $3.58 \times 10^{-1}$ \\
3 & 5.54 & 6.39 & $4.63 \times 10^{-11}$ & $7.82 \times 10^{-}$ \\
4 & 6.37 & 3.80 & $9.47 \times 10^{-1}$ & $5.89 \times 10^{-1}$ \\
5 & 7.43 & 3.17 & $3.53 \times 10^{-1}$ & $7.97 \times 10^{-1}$ \\
6 & 3.61 & 2.18 & $8.54 \times 10^{-5}$ & $2.93 \times 10^{-1}$
\end{tabular}

Another example of the spatial distribution difference in pressure and elevation of network 6 is presented in Figure 7. The hydraulic pressure head surface of the virtual model was subtracted from the hydraulic pressure head surface of the real system. It results clear that the difference in pressure is described by the difference in nodes elevation (i.e. higher elevation results in lower pressure).
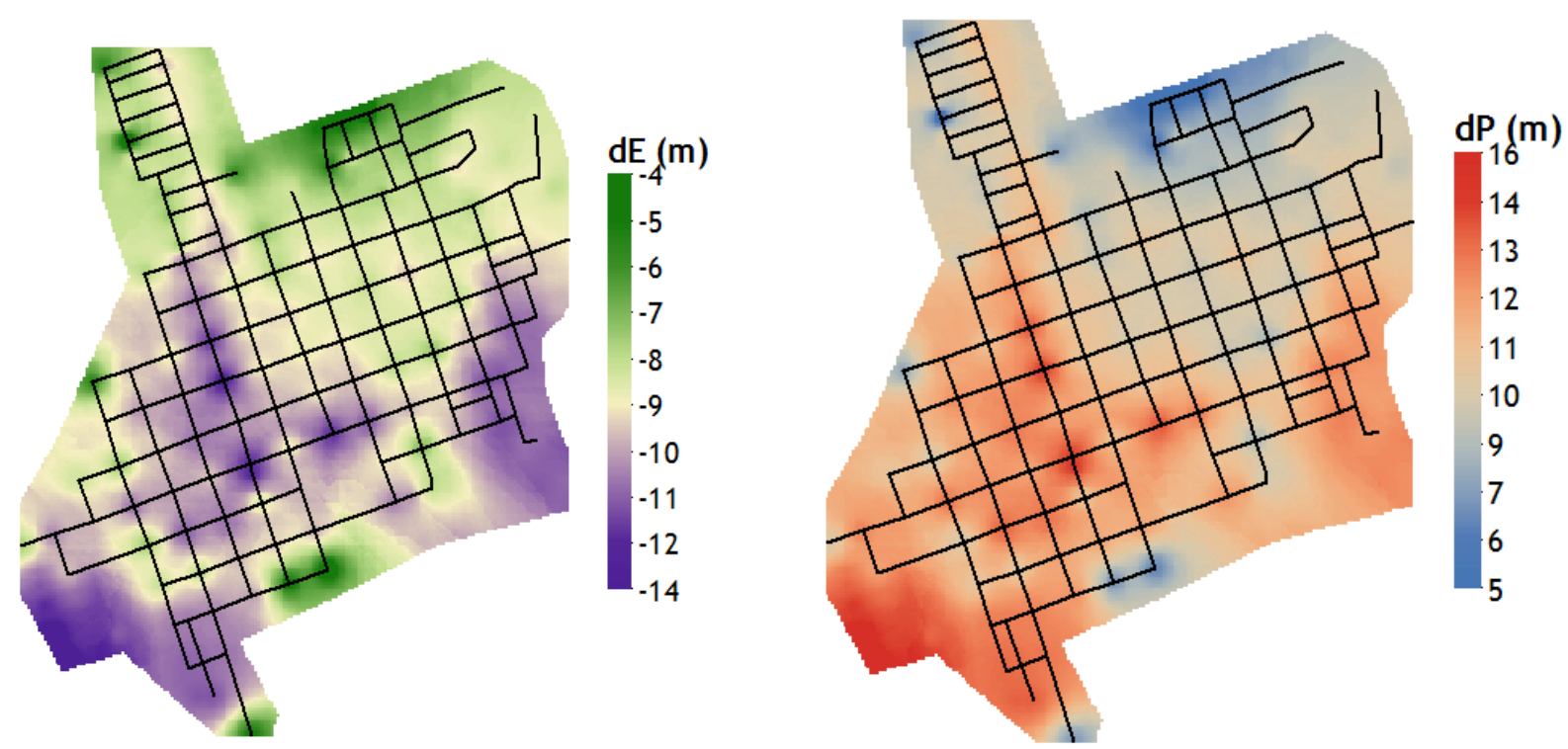

Figure 7. Difference in elevation (dE) and pressure head (dP) between the real and virtual networks

In nearly $50 \%$ of the supply area, the pressure difference between real and virtual networks is below $11 \mathrm{~m}$. Thus, virtual networks present a good performance in terms of pressure. The water age CDF (Figure 8) shows an approximate difference of 8 hours for the $50 \%$ of the network. 

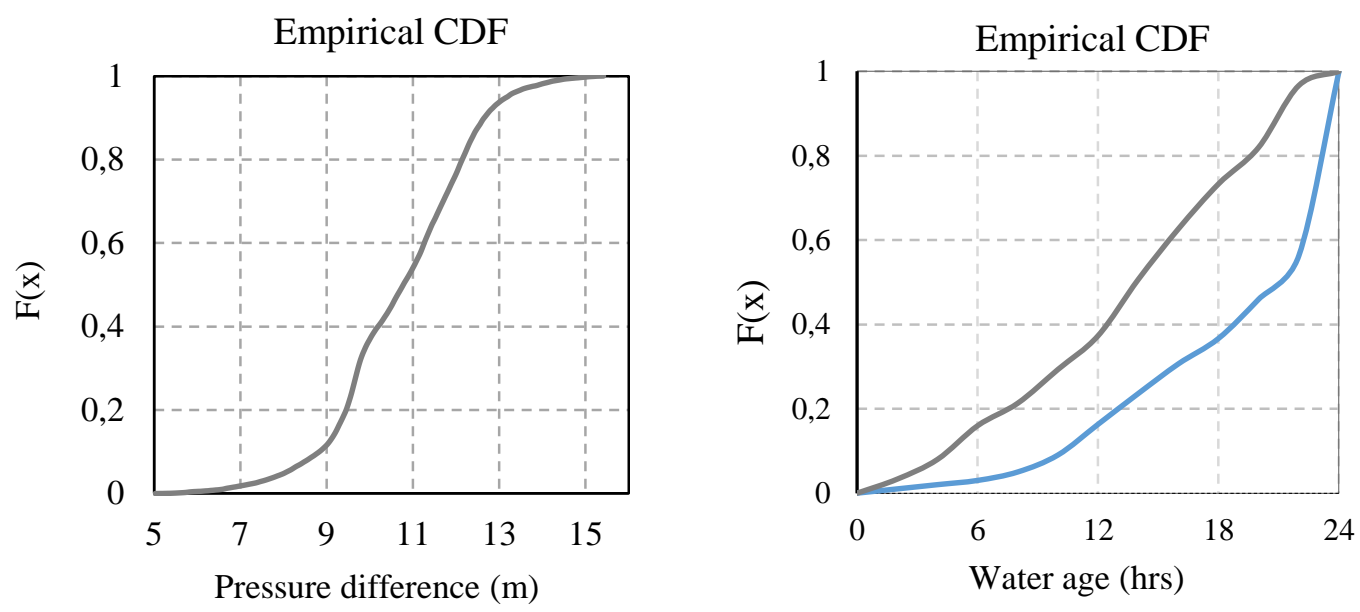

Figure 8. Hydraulic pressure head difference and water quality age CDF

\section{Completing cadastral information}

Considering the already existing and tested WDS hydraulic models for Colombia, a small municipality was chosen for this section. Total demand was thus obtained and the service area was drawn. The expression presented previously was implemented to find the best MPO. Using ArcGIS, the value of the three indicators was computed obtaining a value of $539 \mathrm{~m}$. that resulted in the virtual network presented in Figure 9.

To complete cadastral information, virtual models can be calibrated with minimum required data and from there include known information. If a municipality does not count with a part or a complete register of the system, field measurements can be used to calibrate the synthetic model. To prove this statement, the generated network was calibrated using few input data: four control locations with pressure measures (Figure 9), roughness, list of typical diameters, pattern demand of the network and the reservoir/tank elevation. For this purpose, the information was provided by the available real model. 


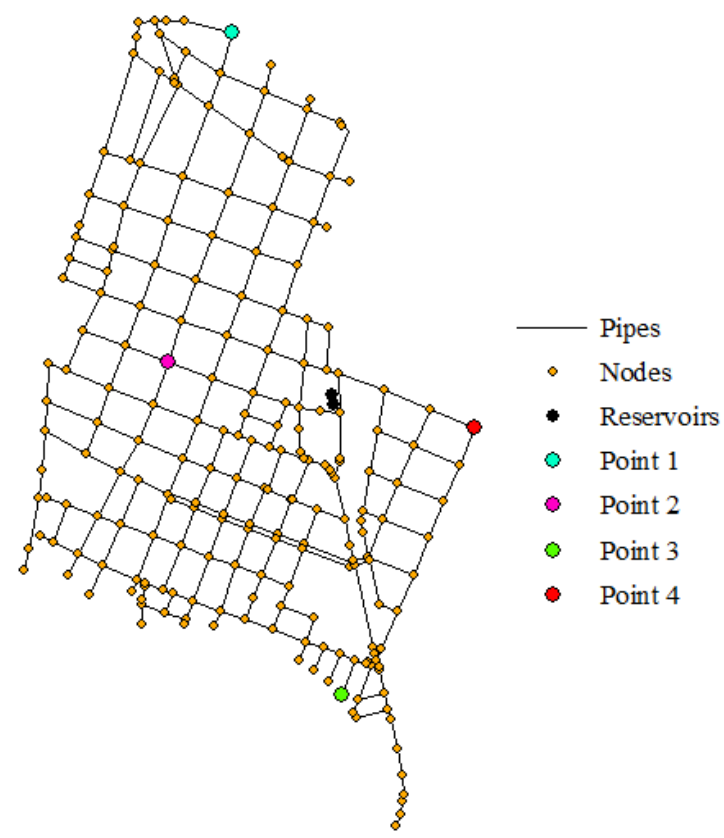

Figure 9. Virtual network and control points

Thus, diameters of the virtual model were calibrated regarding the available values. Considering the DEM used by the software had a resolution of $30 \mathrm{~m}$ x $30 \mathrm{~m}$, and that the municipality had a small geographical extent, the elevation can be inaccurate. For this reason, elevation of the nodes was also calibrated on the iterative process by partitioning the network in zones. The measured and calculated pressure values are presented in Figure 10 with mean differences of $0.3,0.26,0.21$ and 0.03 for locations 1, 2, 3 and 4, respectively. The precision in elevation values for virtual networks is low ( 0.5 m) compared with a higher accuracy for real models, which describe the differences in pressure heads.

a.

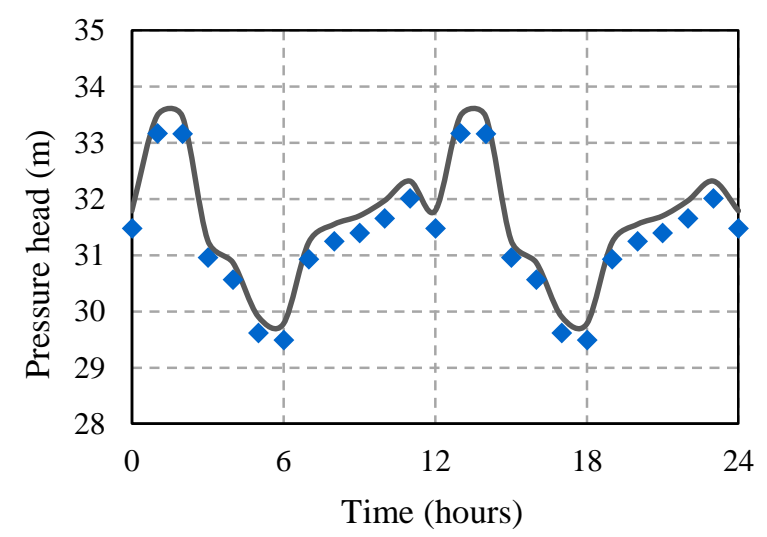

b.

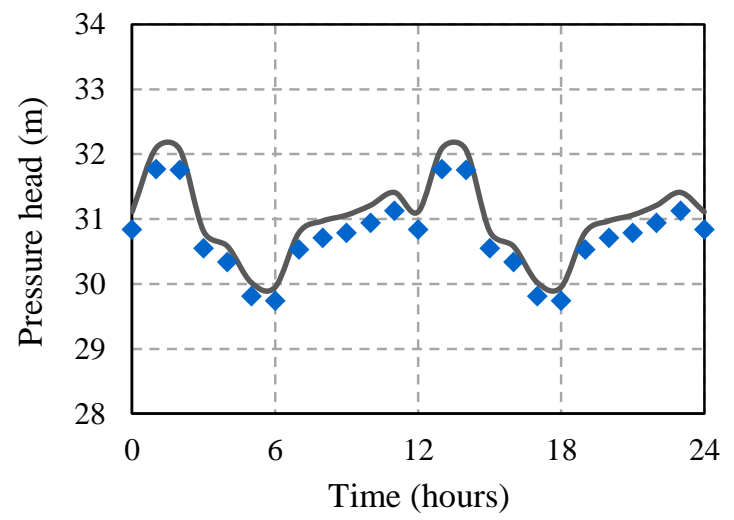


c.

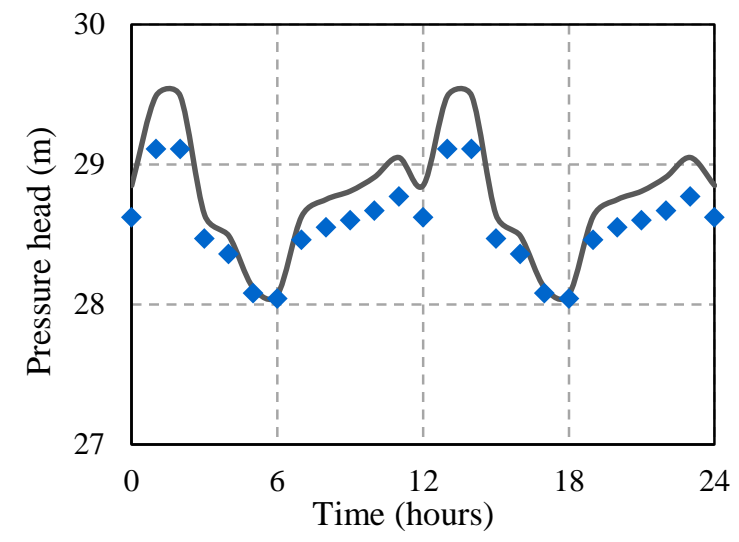

d.

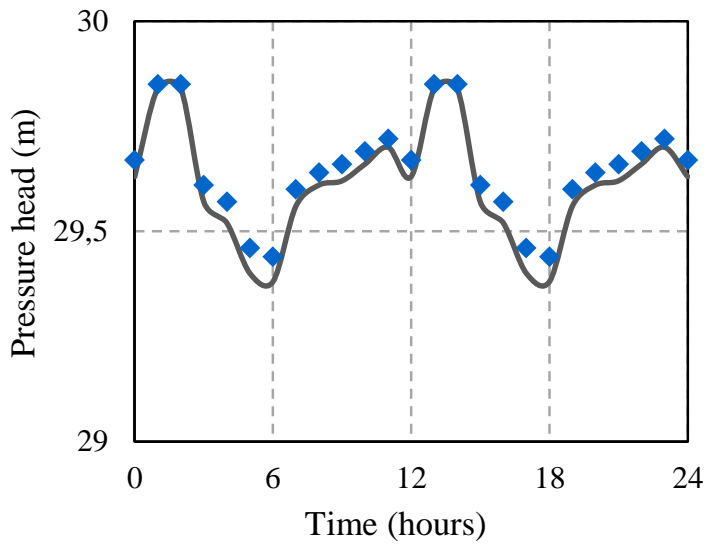

Figure 10. Measured (line) vs. calculated (point) pressure heads for: location 1 (a), location 2(b), location 3 (c) and location 4 (d)

The water distribution network was calibrated based on few input data obtained from the real model and the results show a good agreement between real and calculated pressure heads. Both models are shown in Figure 11. The calibration process and the accuracy of the results become easier with more available data.
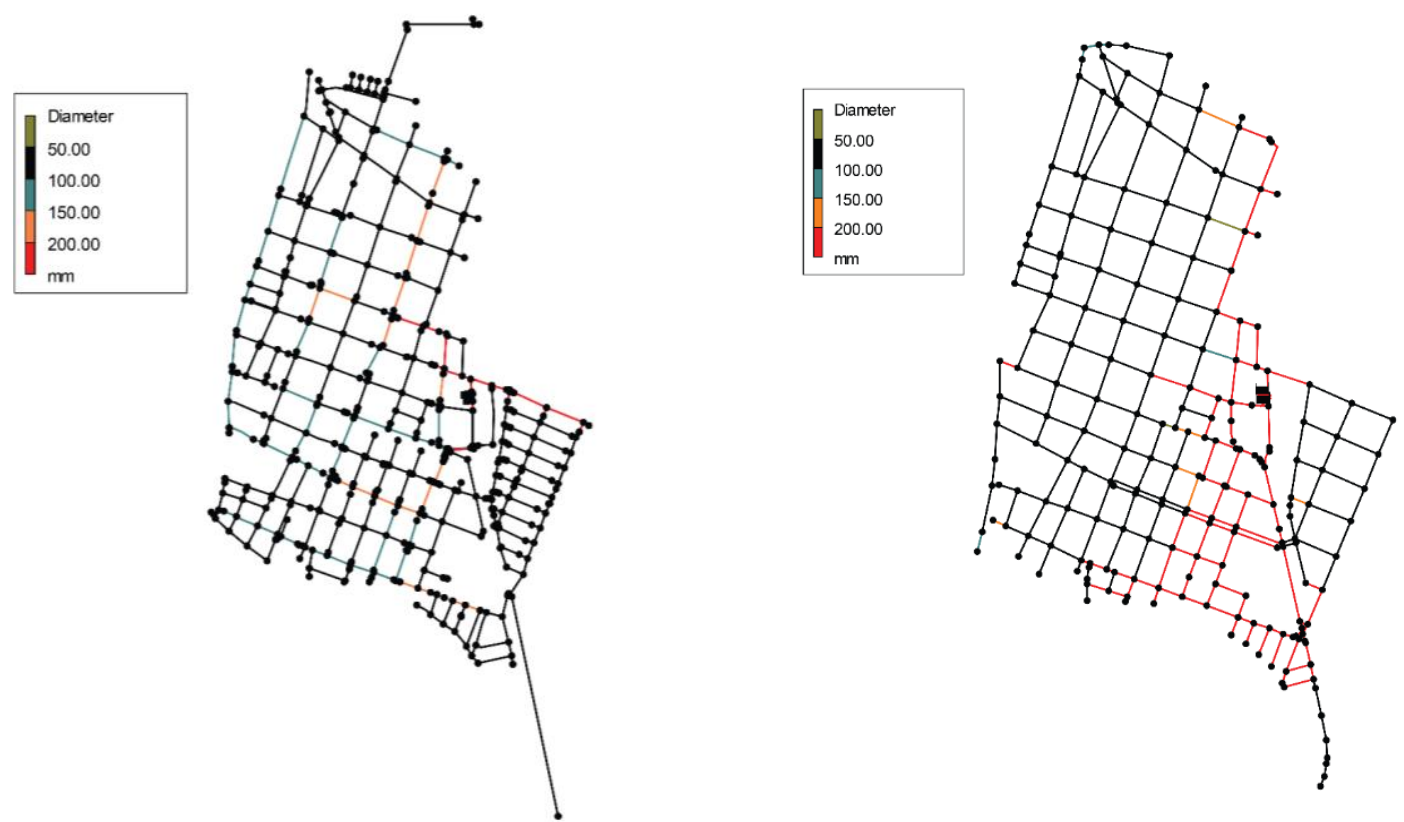

Figure 11. Real and calibrated model 


\section{CONCLUSIONS}

The software DynaVIBe-Web described here enables the generation of virtual networks based on easily accessible street network data. This approach was used to obtain six virtual networks that were compared with the real calibrated models in terms of graph theory and hydraulic indexes. Results are similar for the measures assessed in terms of structure, robustness and connectivity for virtual and real networks, with values within the literature ranges. The synthetic model can mimic the layout characteristics of the real model with a high accuracy. Additionally, resilience index, water age and pressure head surface were analyzed for each pair of sets. Virtual networks exhibit worse working conditions due to the change in tank/reservoir elevation, leading to negative pressures and low resilience values.

Finally, a virtual model was calibrated based on few data input obtained by the real model. Four control points with data assumed as field measures and promising results were obtained for the pressure distribution. Generated models can be calibrated with available information and used with research or management aims without data availability problems. A simplified expression for the MPO calculation was also proposed to facilitate the use of this software for Colombian small municipalities or DMAs in main cities.

\section{REFERENCES}

Araque, D. and Saldarriaga, J. G. (2005). Water Distribution Network Operational Optimization By Maximizing the Pressure Uniformity At Service Nodes, 1-10. https://doi.org/10.1061/40941(247)179

Brumbelow, K., Torres, J., Guikema, S., Bristow, E., and Kanta, L. (2007). Virtual Cities for Water Distribution and Infrastructure System Research. World Environmental and Water Resources Congress 2007, 1-7. http://doi.org/10.1061/40927(243)469

Di Nardo, A., Di Natale, M., Santonastaso, G. F., Tzatchkov, V. G., and Alcocer-Yamanaka, V. H. (2015). Performance indices for water network partitioning and sectorization. Water Science and Technology: Water Supply, 15(3), 499-509. http://doi.org/10.2166/ws.2014.132

Mair, M., Rauch, W., and Sitzenfrei, R. (2014). Spanning tree based algorithm for generating water distribution network sets by using street network data sets (pp. 465-474).

Paez, D., and Filion, Y. (2016). Generation and validation of synthetic WDS case studies using graph theory and reliability indexes. http://doi.org/10.1016/j.seta.2016.12.002

Porse, E., and Lund, J. (2016). Network Analysis and Visualizations of Water Resources Infrastructure in California: Linking Connectivity and Resilience. Journal of Water Resources Planning and Management, 142(1), 04015041. http://doi.org/10.1061/(ASCE)WR.19435452.0000556

Saldarriaga, J. G., Ochoa, S., Rodríguez, D. and Arbeláez, J. (2008). Water Distribution Network Skeletonization Using the Resilience Concept. In Water Distribution System Analysis Conference WDSA2018 (pp. 852-864).

Sitzenfrei, R. (2012). Stochastic Generation of Urban Water Systems for Case Study Analysis. Retrieved from http://www.amazon.de/Stochastic-Generation-Urban-SystemsAnalysis/dp/3902811447/ref=sr_1_1?s=books-intl-de\&ie=UTF8\&qid=1353759872\&sr=1-1

Sitzenfrei, R. (2016). A Review on Network Generator Algorithms for Water Supply Modelling and Application Studies (pp. 505-514). 
Sitzenfrei, R., Möderl, M., and Rauch, W. (2010). WDS Designer- A Tool Algorithmic Generation of Water Distribution Systems Based on GIS Data. Proceedings of the World Environmental and Water Resources Congress 2010: Challenges of Change, 4193-4204. http://doi.org/10.1061/41114(371)427

Todini, E. (2000). Looped water distribution networks design using a resilience index based heuristic approach. Urban Water, 2(2), 115-122. http://doi.org/10.1016/S14620758(00)00049-2

Yazdani, A., and Jeffrey, P. (2011). Complex network analysis of water distribution systems, 1-11. http://doi.org/10.1063/1.3540339 